\title{
Viés de seleção, migração e saúde
}

\author{
Victor Rodrigues de Oliveira*
}

\section{Resumo}

O objetivo do estudo é avaliar o impacto da migração sobre o estado de saúde individual. Esta hipótese proposta por Hull (1979) será testada a partir de três estratégias empíricas: i) um modelo probit ordenado, considerando o impacto da migração por meio de uma variável dummy; ii) um modelo probit multinomial para corrigir o viés de seleção; e iii) o método de estimação semiparamétrico proposto por De Luca e Perotti (2011). Os resultados dos modelos estimados indicaram que o viés de seleção é relevante no caso dos homens, enquanto o modelo que não corrige o viés é o mais adequado para as mulheres. Melhores condições de saúde estão associadas a maiores níveis de educação formal e de renda.

Palavras-chave: Migração. Saúde. Viés de seleção.

* Doutorando do Programa de Pós-Graduação em Economia Aplicada - PPGE/UFRGS. E-mail: victor5491@gmail.com 


\section{Introdução}

Os fluxos migratórios têm impactos sobre o nível de desenvolvimento de uma população, isto é, as migrações são parte integrante de um processo socioeconômico e tem uma regularidade que pode ser observada sob a forma de fluxos, alguns dos quais, devido à sua importância para a dinâmica espacial da economia, assumem caráter estrutural e transformam-se em trajetórias desenhadas de acordo com as necessidades nacionais (BRITO, 2002; LIMA; SIMÕES; OLIVEIRA, 2012).

Conforme Brito (1997) e Lima, Simões e Oliveira (2012), os padrões migratórios podem ser analisados a partir de três grandes ciclos, a saber: i) o do período 1870-1930, caracterizado por migrações internas pouco significativas, resultantes da pequena população brasileira e do papel desempenhado pelas migrações internacionais; ii) o do período 1940-1980, marcado pelas migrações entre a Região Nordeste e Minas Gerais - reservatório de mão de obra - e entre os estados de São Paulo e Rio de Janeiro - devido à expansão industrial e à geração de emprego; e iii) o do período a partir de 1980, em que há um padrão de migração de retorno. Como apontado por Simões e Amaral (2012, p. 554), observa-se recentemente um arrefecimento do processo de metropolização brasileiro, por intermédio de um movimento de interiorização da atividade econômica e de criação de novas centralidades urbanas. O que vem sustentando esse processo é um movimento que pode ser caracterizado por duas vias, isto é, por meio da desconcentração-concentrada da atividade industrial e da expansão das fronteiras agropecuária e mineral.

Vários artigos na literatura internacional - Borjas (1987) e Chiswick (1978), por exemplo - sugerem que os migrantes são indivíduos positivamente selecionados, isto é, têm, em média, melhores características não observáveis que os não migrantes. Esses resultados também são válidos para a economia brasileira - ver, por exemplo, Santos Jr., Ferreira e Menezes-Filho (2005) e Silva e Silveira Neto (2005). Observa-se que os estudos que procuram avaliar os efeitos da migração o fazem sobre os rendimentos dos indivíduos. Isto é, a partir de uma regressão minceriana para o logaritmo do salário contra diversos controles, uma variável dummy é inserida para indicar se o indivíduo é migrante ou não. De forma geral, nota-se que essa variável dummy tem coeficiente positivo e significativo. Ou seja, se após todos os controles usados, essa variável ainda tiver um coeficiente positivo e significativo, essa variável de migração está captando o efeito das características não observáveis e os migrantes são, portanto, positivamente selecionados.

Entretanto, Maddala (1984) demonstra que a inclusão dessa variável não é suficiente para corrigir o possível viés de seleção existente na amostra. Neste caso, 
são utilizados os métodos baseados em Heckman (1979) e recentemente a proposta de Dahl (2002), que supera os problemas de controle do viés de seleção em ambientes de escolha múltipla (mais de duas opções), empregando um modelo semiparamétrico. O estudo de Ribeiro e Bastos (2004) propõe a estimativa dos retornos à educação para os estados brasileiros, corrigindo o problema de viés por seleção gerado pela migração, dentro de um modelo de Roy de salários. ${ }^{1}$ A correção da seleção, empregando a técnica desenvolvida por Dahl (2002) indicou que parece existir viés de seleção dos indivíduos nas estimativas das equações de salários, viés esse que não é corrigido por meio de uma variável dummy para migração. Verificou-se ainda que os retornos à educação crescem à medida que o nível educacional aumenta e que as diferenças regionais dos retornos aumentam com a correção das estimativas.

Todavia, pouca atenção é dedicada à relação entre saúde e migração. Essa pode ser dividida em duas abordagens:

I- os estudos que procuram avaliar a condição de acesso dos migrantes aos serviços de saúde;

II- os estudos que investigam o impacto do processo migratório sobre a saúde individual.

A literatura que estuda o primeiro efeito discute o direito ao acesso aos serviços de saúde. Como debatido por Bäckström (2010), os principais obstáculos no acesso aos cuidados de saúde evoluem com o passar dos anos decorridos sem autorização de estadia no país de acolhimento. A situação irregular reflete-se em todos os domínios da vida de um imigrante: emprego, habitação, serviços sociais, saúde, educação, justiça, tornando-se um ciclo vicioso que aumenta as situações de vulnerabilidade, de pobreza e de exclusão social. Muitos estudos da Organização Mundial da Saúde (OMS) (WHO, 2009; WHO, 2010) avaliam essa questão, tais como Migration and health in the European Union (2009) e How health systems can address health inequities linked to migration and ethnicity (2010).

Por outro lado, a literatura sobre migração e saúde individual é escassa. De acordo com Hull (1979), o estado de saúde de um indivíduo pode afetar as decisões de migração (a hipótese de seleção de saúde) e a migração pode afetar a saúde das pessoas que se deslocam (devido ao rompimento da vida individual e à adaptação a novos ambientes e devido a melhores serviços de saúde na região de destino). Do ponto de vista econômico, a compreensão do efeito líquido da migração sobre o estado de saúde é importante por várias razões. Em termos de crescimento econômico esse deslocamento pode ter consequências financeiras (privadas e públicas) significativas de longo prazo para as regiões de origem e de destino. Além disso, os 
benefícios do aumento da produtividade e do maior crescimento econômico, devido aos fluxos migratórios, poderiam ser reduzidos por cuidados de saúde a longo prazo e custos de assistência social, em caso de deterioração do estado de saúde.

Como observado por Lu (2008), a grande maioria da literatura existente sobre migração e saúde compara o status de saúde dos imigrantes com o status da população dos países de destino, em vez de avaliá-lo nas regiões de origem. De acordo com essa literatura, os imigrantes são geralmente mais saudáveis do que as populações não migrantes. No entanto, essa vantagem deteriora-se ao longo do tempo devido às condições de vida adversas na nova região (ANSON, 2004; FERANIL, 2005; MARMOT; ADELSTEIN; BULUSU, 1984a, 1984b; PALLONI; MORENOFF, 2001). Este fenômeno tem sido referido na literatura como paradoxo epidemiológico.

Uma série de estudos examinam os efeitos da migração sobre a saúde no curto prazo. Quando comparado com as populações de origem, Kanaiaupuni e Donato (1999) constataram que a mortalidade infantil diminuiu com o aumento do fluxo de remessas para cinco estados mexicanos que tradicionalmente enviaram migrantes para os Estados Unidos. Frank e Hummer (2002) observam que a incidência de baixo peso ao nascer é reduzida em domicílios com migrantes. Em suma, verifica-se que os migrantes têm melhor saúde e menor mortalidade do que no local de nascimento devido à seleção dos candidatos mais aptos para a imigração. Gibson e McKenzie (2007), por sua vez, mostraram que os migrantes de Tonga e Nova Zelândia apresentaram um status sanitário inferior àqueles no grupo de controle que não migraram.

O principal estudo a respeito desta temática é o de Atella e Deb (2013). A partir de um modelo de misturas finitas ${ }^{2}$ e dos dados do Multiscopo Survey para o período 1950 a 1970, os autores consideram um possível viés de seleção decorrente da não observação do estado de saúde individual anteriormente à migração. Os resultados encontrados indicaram que há efeitos de longo prazo da migração sobre o estado de saúde individual para as mulheres residentes na área rural. Apesar desses resultados, não foram encontradas evidências de viés de seleção.

A partir do exposto, o objetivo deste estudo é avaliar os efeitos da migração sobre a condição de saúde individual para os estados brasileiros no ano de 2008. Para verificar a existência de viés de seleção serão utilizadas duas estratégias empíricas, a saber: i) um modelo multinomial para avaliar o status de saúde autorreportada e, posteriormente, um modelo probit ordenado para investigar o efeito da migração sobre a condição de saúde; e ii) um modelo probit ordenado, que corrige o viés por meio de uma abordagem semiparamétrica, como proposto por De Luca e Perotti (2011). 
Além dessa introdução, o estudo será organizado como segue. Na próxima seção, serão apresentados os modelos empíricos utilizados para testar o impacto da migração sobre a condição de saúde individual e a fonte de dados utilizada. $\mathrm{Na}$ seção 3, serão apresentadas e discutidas as evidências dos modelos paramétrico e semiparamétrico. Por fim, serão abordadas as conclusões.

\section{Modelos empíricos e fonte de dados}

A estratégia empírica utilizada consiste de duas abordagens, que serão apresentados nesta seção. Em primeiro lugar, será descrito o modelo que corrige o viés de seleção por meio de um modelo multinomial. Em segundo lugar, será discutida a abordagem semiparamétrica proposta por De Luca e Perotti (2011).

\subsection{Correção do viés de seleção por meio de um modelo multinomial}

Considere o seguinte modelo: $\quad \boldsymbol{S}_{i}=\boldsymbol{x}_{i}^{\prime} \beta+\boldsymbol{p}_{i}^{\prime} \lambda$

$$
S_{i}=x_{i}^{\prime} \beta
$$

onde $\boldsymbol{S}_{i}$ é a condição de saúde do indivíduo $i$ no estado onde reside, $\boldsymbol{x}_{i}^{\prime}$ é um vetor de covariadas que inclui uma dummy para capturar a condição de migração e $\beta$ é um vetor de parâmetros a ser estimado. O problema para estimação da equação (1) é que se observa a condição de saúde apenas no estado em que a pessoa se encontra. Não se sabe sua condição de saúde no estado de origem. Isso é um problema, pois a decisão de migrar do estado $j$ para o estado $k$, ou seja, a decisão que faz com que $S$ seja observado depende das condições de saúde em $S_{k}$ e $S_{k j}$, isto é, $E[u \mid \mathrm{S}$ observado $] \neq 0$, criando um viés de seleção nas estimativas dos coeficientes (MADDALA, 1984). Assim, é necessário incluir um termo em (1) para corrigir o viés de seleção, como segue:

$$
\boldsymbol{S}_{i}=\boldsymbol{x}_{i}^{\prime} \beta+\boldsymbol{p}_{i}^{\prime} \lambda
$$

em que $\boldsymbol{p}_{i}$ é a probabilidade do indivíduo $i$ migrar do estado $j$ para o estado $k$ e o termo $\lambda$ corrige $o$ viés de seleção. Para obter a probabilidade de migração a literatura empírica aponta diversos métodos. O método mais usual é o Heckit com duas regiões apenas, que representa um problema simples de escolha binária. 
Entretanto, essa alternativa é viável apenas se tivermos duas opções, por exemplo, migrar ou não migrar, usando um probit simples para calcular a inversa da razão de Mills (HECKMAN, 1979).

Dahl (2002), por sua vez, propõe um método semiparamétrico para se obterem as probabilidades de migração. $O$ termo para corrigir o viés de seleção é desconhecido, mas, por ser univariado, pode ser facilmente aproximado por um polinômio de ordem satisfatória. Dessa forma, a estimação tem por hipótese índice único, single index (POWELL, 1997). Todavia, Dahl (2002) simplifica o número de funções $\lambda$ a serem estimadas, considerando que as essas são iguais para os migrantes independentemente da origem.

Neste estudo, adotou-se um modelo probit multinomial para determinar a probabilidade de migrar do estado $j$ para o estado $k$. Essas probabilidades são substituídas em (2). Conforme Cameron e Trivedi (2005), esse é um modelo com $m$ -alternativas, que associa a seguinte função de utilidade:

$$
\begin{array}{rlr}
U_{j} & =V_{j}+\varepsilon_{j}, & j=1,2, \ldots, m \\
& =\mathbf{z}_{j}^{\prime} \gamma+\varepsilon_{j}, & j=1,2, \ldots, m
\end{array}
$$

em que $\varepsilon \sim \mathcal{N}[\mathbf{0}, \boldsymbol{\Sigma}], V_{j}$ é o estado de destino do indivíduo, $\boldsymbol{Z}$ é um vetor de covariadas e $\gamma$ um vetor de parâmetros a ser estimado. Em decorrência do tamanho da amostra e do número de covariadas há uma grande exigência computacional e, por isso, adotou-se o algoritmo recursivo de suavização $G H K^{3}$ (GEWEKE, 1992; HAJIVASSILIOU; MCFADDEN, 1994; KEANE, 1994). Dessa forma, a primeira etapa consiste em estimar (3) como um dos argumentos de (2). Na estimação dos erros-padrão das probabilidades de migração considerou-se que essa decisão envolve, além de atributos individuais, decisões no âmbito da família - o tamanho da família e a estrutura familiar. Por conseguinte, inclui-se na análise a noção de clusters com o objetivo de corrigir a precisão das probabilidades.

Em seguida, estimou-se um modelo probit ordenado, pois a variável dependente apresenta uma ordem natural (WOOLDRIDGE, 2002; CAMERON; TRIVEDI, 2005). Assumindo $\boldsymbol{S}_{i}$ como uma variável latente, tem-se:

$$
\boldsymbol{S}_{i}=\boldsymbol{x}_{i}^{\prime} \beta+u_{i}
$$

em que $\boldsymbol{x}_{i}^{\prime}$ inclui a probabilidade do indivíduo $i$ migrar do estado $j$ para o estado $k$ e as demais covariadas. Nesse modelo, por exemplo, para um valor baixo de $\boldsymbol{S}_{i}$ a condição de saúde é péssima, para $S_{i}>a_{1}$ a condição de saúde é ruim e assim por diante. Para um modelo ordenado com $m$-alternativas define-se: 


$$
y_{i}=j \text { se } \alpha_{j-1}<S_{i}<\alpha_{j}
$$

em que $\alpha_{0}=-\infty$ e $\alpha_{m}=\infty$. Dessa forma,

$$
\begin{aligned}
\operatorname{Pr}\left[\boldsymbol{y}_{i}=j\right] & =\operatorname{Pr}\left[\alpha_{j-1}<\boldsymbol{S}_{i}<\alpha_{j}\right] \\
& =\operatorname{Pr}\left[\alpha_{j-1}<\boldsymbol{x}_{i}^{\prime} \beta+u_{i}<\alpha_{j}\right] \\
& =\operatorname{Pr}\left[\alpha_{j-1}-\boldsymbol{x}_{i}^{\prime} \beta<u_{i} \leq \alpha_{j}-\boldsymbol{x}_{i}^{\prime} \beta\right] \\
& =F\left(\alpha_{j}-\boldsymbol{x}_{i}^{\prime} \beta\right)-F\left(\alpha_{j-1}-\boldsymbol{x}_{i}^{\prime} \beta\right)
\end{aligned}
$$

em que $F$ é a função de distribuição acumulada de $u_{i}$. Os parâmetros $\beta$ podem ser interpretados diretamente como determinantes do aumento ou não da variável latente $\boldsymbol{S}_{i}$.

\subsection{Correção do viés de seleção por meio de um modelo semiparamétrico}

Nesta abordagem, a seleção da amostra ocorre por meio de uma decisão: migrar ou não. Essa escolha é semelhante a um modelo que empregue o Heckit, porém considera que a probabilidade de migrar ou não segue um processo não paramétrico. Para compreender o método proposto por De Luca e Perotti (2011) suponha a seguinte estrutura:

$$
\begin{aligned}
& \boldsymbol{Y}_{j}^{*}=\beta_{j}^{\prime} \boldsymbol{X}_{j}+u_{j}, \quad j=1,2 \\
& Y_{1}=I\left(Y_{1}^{*} \geq 0\right) \\
& Y_{2}=\sum_{h=0}^{H} h I\left(\alpha_{h}<Y_{2}^{*} \leq \alpha_{h+1}\right)
\end{aligned}
$$

em que $Y_{1}^{*}$ e $Y_{2}^{*}$ representam as variáveis latentes para o processo de seleção amostral, $I(A)$ denota uma função indicadora do evento $A$ e $u_{j}$ são os termos de erro. A variável latente $Y_{1}^{*}$ está relacionada ao indicador binário $Y_{1}$ por meio da regra (8). Para $Y_{2}^{*}$ a regra está definida em (9). Relaxando os pressupostos sobre a distribuição de $u_{1}$ e $u_{2}$, a especificação semiparamétrica do modelo (7)-(9) apresenta o seguinte conjunto de probabilidades condicionais: 


$$
\begin{aligned}
\pi_{0}\left(\beta_{1}, \beta_{2}, \alpha\right)= & F_{1}\left(-\beta_{1}^{\prime} \boldsymbol{X}_{1}\right) \\
\pi_{1 h}\left(\beta_{1}, \beta_{2}, \alpha\right)= & {\left[F_{2}\left(\alpha_{h+1}-\beta_{2}^{\prime} \boldsymbol{X}_{2}\right)-F\left(-\beta_{1}^{\prime} \boldsymbol{X}_{1}, \alpha_{h+1}-\beta_{2}^{\prime} \boldsymbol{X}_{2}\right)\right] } \\
- & {\left[F_{2}\left(\alpha_{h}-\beta_{2}^{\prime} \boldsymbol{X}_{2}\right)-F\left(-\beta_{1}^{\prime} \boldsymbol{X}_{1}, \alpha_{h}-\beta_{2}^{\prime} \boldsymbol{X}_{2}\right)\right] }
\end{aligned}
$$

em que $F_{1}, F_{2}$ e $F$ denotam, respectivamente, as funções de distribuição marginal desconhecidas de $u_{1}$ e de $u_{2}$ e a função de distribuição conjunta.

Gallant e Nychka (1987) demonstram que a densidade conjunta desconhecida de $f$ pode ser aproximada por uma expansão polinomial de Hermite como segue:

$$
f^{*}\left(u_{1}, u_{2} ; \gamma\right)=\frac{1}{\psi_{R}(\gamma)} \tau_{R}\left(u_{1}, u_{2} ; \gamma\right)^{2} \phi\left(u_{1}\right) \phi\left(u_{2}\right)
$$

em que $\tau_{R}\left(u_{1}, u_{2} ; \gamma\right)$ é um polinômio de ordem $R=\left(R_{1}, R_{2}\right)$ em $u_{1}$ e $u_{2}, \gamma$ é um vetor dos parâmetros desconhecidos, $\phi$ é a função de densidade normal padrão e $\psi_{R}(\gamma)$ é uma normalização para garantir que $f^{*}$ seja uma função de densidade.

De Luca (2008) mostra que a função de distribuição conjunta de $u_{1}$ e de $u_{2}$ pode ser aproximada por meio da integração de (11), resultando em:

$$
\begin{aligned}
F^{*}\left(u_{1}, u_{2} ; \gamma\right)= & \Phi\left(u_{1}\right) \Phi\left(u_{2}\right)+\frac{1}{\psi_{R}(\gamma)} A_{12}^{*}\left(u_{1}, u_{2} ; \gamma\right) \phi\left(u_{1}\right) \phi\left(u_{2}\right) \\
& -\frac{1}{\psi_{R}(\gamma)} A_{1}^{*}\left(u_{1} ; \gamma\right) \Phi\left(u_{2}\right) \phi\left(u_{1}\right)-\frac{1}{\psi_{R}(\gamma)} A_{2}^{*}\left(u_{2} ; \gamma\right) \Phi\left(u_{1}\right) \phi\left(u_{2}\right)
\end{aligned}
$$

em que $A_{12}^{*}\left(u_{1}, u_{2} ; \gamma\right), A_{1}^{*}\left(u_{1} ; \gamma\right)$ e $A_{2}^{*}\left(u_{2} ; \gamma\right)$ são polinômios em $u_{1}$ e em $u_{2}$. De forma semelhante, as distribuições marginais de $u_{1}$ e $u_{2}$ são, respectivamente:

$$
\left\{\begin{array}{l}
F_{1}^{*}\left(u_{1} ; \gamma\right)=\Phi\left(u_{1}\right)-\frac{1}{\psi_{R}(\gamma)} A_{1}^{*}\left(u_{1} ; \gamma\right) \phi\left(u_{1}\right) \\
F_{2}^{*}\left(u_{1} ; \gamma\right)=\Phi\left(u_{2}\right)-\frac{1}{\psi_{R}(\gamma)} A_{2}^{*}\left(u_{2} ; \gamma\right) \phi\left(u_{2}\right)
\end{array}\right.
$$

Considerando que a função de pseudoverissimilhança é dada por:

$$
L(\boldsymbol{\theta})=\sum_{i=1}^{n}\left[\left(1-Y_{1 i}\right) \ln \pi_{0 i}(\boldsymbol{\theta})+\sum_{h=0}^{H} Y_{1 i} I\left(Y_{2 i}=h\right) \ln \pi_{1 h i}(\boldsymbol{\theta})\right]
$$

em que $\boldsymbol{\theta}$ é o vetor de parâmetros a ser estimado, o estimador semiparamétrico é obtido maximizando-se (14) com as probabilidades especificadas em (10). Para o modelo ser identificado $\alpha_{1}$ é fixo. 


\subsection{Base de dados e tratamento da amostra}

A base de dados utilizada neste estudo são os microdados oriundos da Pesquisa Nacional por Amostra de Domicílios (PNAD) realizada pelo Instituto Brasileiro de Geografia e Estatística (IBGE). A PNAD é uma pesquisa por amostragem probabilística de domicílios, realizada em todo o território nacional, a qual apresenta um questionário de caráter permanente, envolvendo perguntas relativas a características domiciliares e pessoais e um de caráter suplementar, em que se investiga características socioeconômicas e demográficas. A análise incorporou a estrutura do plano amostral da PNAD, uma vez que as estimativas dos erros-padrão são influenciadas pelo processo de conglomeração e de estratificação e pelos pesos de cada unidade amostrada (NASCIMENTO SILVA; PESSOA; LILA, 2002).

Conforme recomendações das Nações Unidas, o conceito de migrante pode ser apresentado em duas visões: migrante acumulado e migrante de curto prazo. $\mathrm{O}$ migrante acumulado é aquele que na data da entrevista garantiu residir em um estado diferente do seu estado de nascimento. Por outro lado, o migrante de curto prazo é aquele que, na data da pesquisa, declarou ter até quatro ou até nove anos de domicílio em um estado que não é o seu local de nascimento. Este estudo adotou o primeiro conceito.

As informações foram obtidas para o ano de 2008 e apresenta os resultados do levantamento suplementar de saúde. A Pesquisa Suplementar de Saúde (PSS) compreendeu entre os tópicos pesquisados os seguintes temas: condição de saúde, morbidade, cobertura de plano de saúde, acesso a serviço de saúde, utilização de serviços de saúde, internação, atendimento de urgência no domicílio, violência, acidente de trânsito e sedentarismo, entre outros. A principal variável de interesse, a condição de saúde individual, representa uma escala com cinco valores, a saber: 1 - muito ruim; 2 - ruim; 3 - regular; 4 - bom; e 5 - muito bom.

A análise foi realizada separando-se os indivíduos por gênero. Como enfatizado por Van Der Vem e Ellis (2000), a demanda das mulheres por serviços médicos geralmente é mais elevada do que a dos homens na idade adulta, fenômeno associado ao período fértil feminino e também à maior percepção feminina e preocupação com sua saúde. Na velhice, a demanda masculina ultrapassa a feminina, fato relacionado com o desgaste dos homens no trabalho, levando-os a maior incidência de doenças nessa fase da vida e, geralmente, à morte com idade menos avançada do que as mulheres. Desta forma, essa procura é refletida na avaliação das condições de saúde. 
As estatísticas descritivas da amostra estão na Tabela 1 em anexo. As variáveis utilizadas estão apresentadas na Tabela 2.

Tabela 2 - Descrição das variáveis utilizadas nas regressões

\begin{tabular}{|c|c|}
\hline Variável & Descrição (conteúdo das variáveis) \\
\hline Estado de saúde & 1 - muito ruim*; 2 - ruim; 3 - regular; 4 - bom; 5 - muito bom. \\
\hline Idade & Representada pela idade declarada do indivíduo. \\
\hline Cor & $\begin{array}{l}\text { Representada por cinco variáveis dummy: } 1 \text { - pessoas que se declaram brancas*; } \\
2 \text { - pessoas que se declaram pretas; } 3 \text { - pessoas que se declaram amarelas; } 4 \text { - } \\
\text { pessoas que se declaram parda; } 5 \text { - pessoas que se declaram indígenas. }\end{array}$ \\
\hline Educação & $\begin{array}{l}\text { Representada por quatro variáveis dummy: } 1 \text { - indivíduos sem instrução e menos } \\
\text { de um ano de estudo*; } 2 \text { - indivíduos com um ano a quatro anos de estudo; } 3 \text { - in- } \\
\text { divíduos com cinco a oito anos de estudo; } 4 \text { - indivíduos com nove anos ou mais } \\
\text { anos de estudo. }\end{array}$ \\
\hline Ocupação & $\begin{array}{l}\text { Composta por seis variáveis dummy: } 1 \text { - indivíduos desocupados*; } 2 \text { - indivíduos } \\
\text { com carteira assinada; } 3 \text { - indivíduos empregadores; } 4 \text { - indivíduos que trabalham } \\
\text { por conta própria; } 5 \text { - indivíduos que constroem para consumo e uso próprio; } 6 \text { - de- } \\
\text { mais indivíduos com carteira assinada. }\end{array}$ \\
\hline Renda monetária & $\begin{array}{l}\text { Representada por variáveis dummy que dividem os indivíduos de acordo com o ren- } \\
\text { dimento declarado, em frações de salário mínimo: } 1 \text { - sem renda*; } 2 \text { - até um sa- } \\
\text { lário; } 3 \text { - entre dois e três salários; } 4 \text { - entre três e quatro salários; } 5 \text { - entre quatro } \\
\text { e cinco salários; } 6 \text { - entre cinco e seis salários; } 7 \text { - mais de seis salários mínimos. }\end{array}$ \\
\hline Bens de consumo & $\begin{array}{l}\text { O acesso a bens de consumo duráveis foi avaliado pela posse de televisão, de } \\
\text { fogão, de rádio, de geladeira, de máquina de lavar e de computador. Para tanto, a } \\
\text { elaboração de um índice recorreu à análise fatorial com a extração de dois compo- } \\
\text { nentes principais. }\end{array}$ \\
\hline Saneamento & $\begin{array}{l}\text { Foram utilizados três indicadores: i) tem água canalizada em pelo menos um cômo- } \\
\text { do do domicílio; ii) tem banheiro ou sanitário no domicílio; e iii) se o lixo domiciliar é } \\
\text { coletado. Esses indicadores foram utilizados para construir um índice por intemédio } \\
\text { de análise fatorial com a extração de dois componentes principais. }\end{array}$ \\
\hline Variáveis geográficas & $\begin{array}{l}\text { Uma variável dummy para área urbana, uma variável dummy para região metropoli- } \\
\text { tana e dummies para os estados. }\end{array}$ \\
\hline Tamanho da família & Número de membros que compõem a família. \\
\hline
\end{tabular}

Fonte: elaboração própria a partir de dados da PNAD de 2008.

Nota: * Categoria de referência/controle.

Na próxima seção serão apresentados e discutidos os resultados do modelo estimado, comparando-se os modelos com correção e sem correção da variável migração.

\section{Resultados do modelo}

A Tabela 3 apresenta os resultados dos modelos sem e com a correção do viés de seleção. Como exposto anteriormente, adotou-se a hipótese de Van Der Vem e Ellis (2000), que propõe que os cuidados de saúde diferem entre homens e mulheres. 
Assim, há diferenças na avaliação das condições de saúde individual e, portanto, a migração impacta de forma distinta entre homens e mulheres, como defendido por Atella e Deb (2013). A partir disso, serão apresentadas e discutidas as estimativas dos coeficientes. O resultado do teste de Wald mostrou-se estatisticamente significativo a $1 \%$ para os três modelos estimados. Para o modelo com correção semiparamétrica, estimou-se o polinômio, $\tau_{R}$, de ordem 3 .

I- Idade: considerou-se o impacto da idade para capturar de forma mais adequada a distribuição da variável em relação à condição de saúde. Observa-se que essa apresentou um sinal negativo e é estatisticamente significativa a $1 \%$. Assim, indivíduos mais velhos tendem, em média, a reportarem as piores condições de saúde e essa ocorre a taxas cada vez maiores, como exposto pela variável idade ao quadrado. Quando se observa a relação dessa com a variável de interesse, mas diferenciando-se por tipo de indivíduo, homem ou mulher, percebe-se que os homens mais velhos apresentam piores condições de saúde (incidência de doenças e condições de mobilidade, dentre outras), que se traduzem em estados de saúde com menor status, ou seja, avaliam sua saúde como de menor "qualidade". Esse resultado corrobora, em parte, o argumento apresentado por Van Der Vem e Ellis (2000) de que a diferença de gênero entre os indivíduos é fundamental para explicar sua qualidade de vida e, portanto, sua necessidade de atenção médica básica e especializada, de forma a atender suas novas "demandas" por saúde.

II- Cor: os resultados dos coeficientes devem ser interpretados em relação à variável omitida, nesse caso os indivíduos que se autodeclararam brancos. Para os indivíduos que se autodeclararam pretos, pardos e indígenas nota-se que os parâmetros estimados apresentaram sinal negativo e foram estatisticamente significativos. Por sua vez, para os homens que se autodeclararam amarelos essa variável não foi estatisticamente significativa. A variável cor procura "capturar" de forma indireta a desigualdade no acesso a serviços de saúde, que aumentariam a depreciação do estoque de saúde individual.

III- Anos de estudo: os resultados dos coeficientes devem ser interpretados em relação à variável omitida, neste caso os indivíduos sem instrução e com menos de um ano de estudo. Para o primeiro nível de escolarização ( 1 a 4 anos de estudo) e o segundo nível (de 5 a 8 anos), o coeficiente é significativo e com sinal positivo, conforme o esperado. Para o terceiro nível, que representa os indivíduos que ao menos começou a cursar o ensino médio, o coeficiente é positivo e de maior magnitude. Esses resultados indicam que a saúde é positivamente relacionada com a educação, isto é, os indivíduos mais educados consomem bens que irão diminuir a taxa de depreciação do 
seu estoque de saúde, tornando-o mais saudável. Além disso, uma vez que uma menor depreciação deve ser reposta, indivíduos com melhor educação apresentam menor necessidade de investimento para manutenção de seu estoque (GROSSMAN, 1972). Assim, percebe-se a noção de retornos crescentes à educação formal. Isto é, quanto maior o número de anos de estudo formal, melhores serão os estados de saúde reportados. A Figura 1, referente ao modelo para homens com correção do viés de seleção por intermédio do modelo multinomial, demonstra esse efeito. ${ }^{4}$

Figura 1 - Relação entre probabilidade dos estados de saúde e anos de estudo
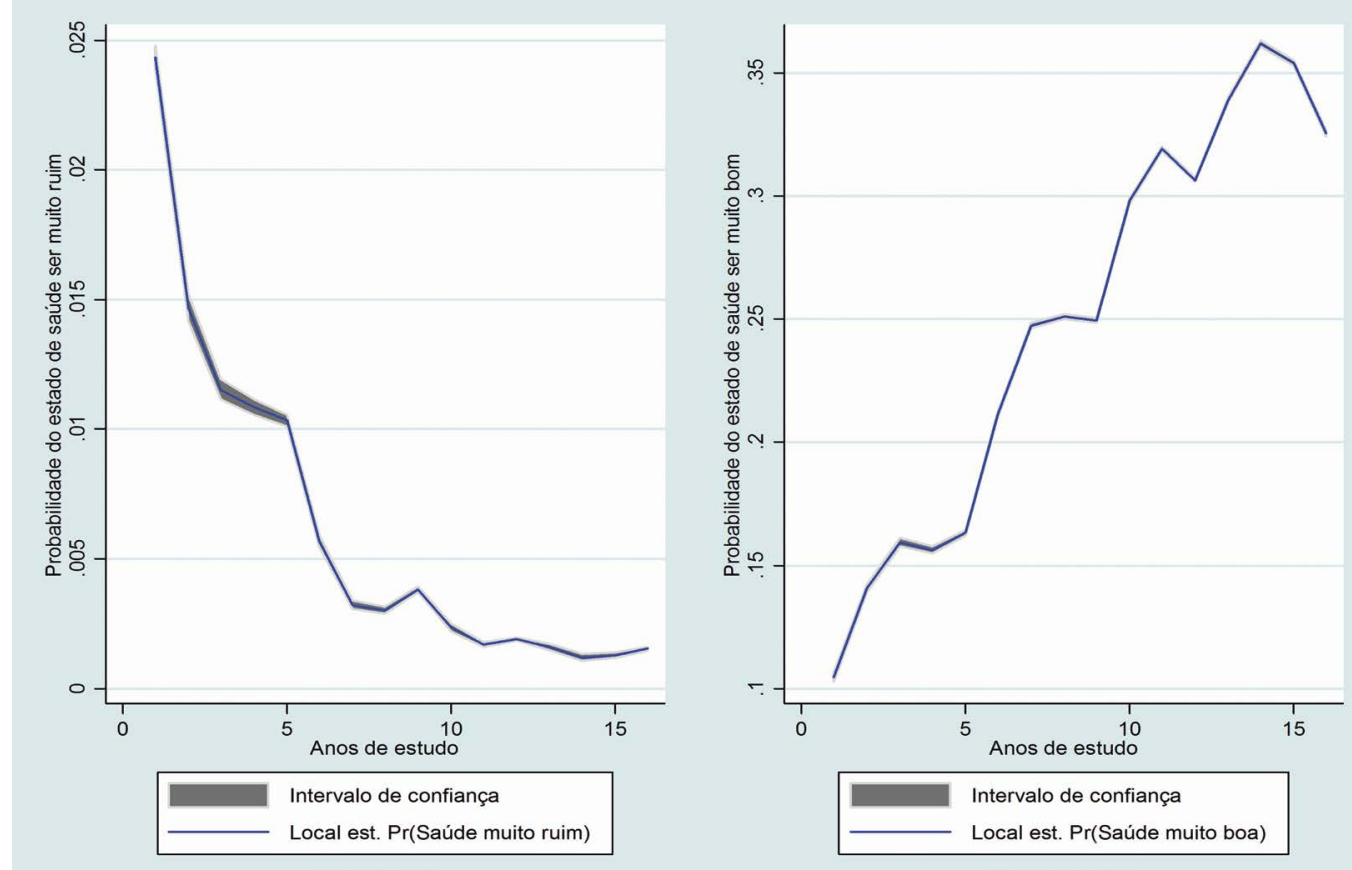

Fonte: elaborado pelos autores.

IV- Ocupação: os resultados deste grupo de variáveis foram todos estatisticamente significativos e nota-se que qualquer ocupação no mercado de trabalho (com exceção das mulheres trabalhadoras na construção para o próprio uso no modelo 2 e homens com carteira assinada no modelo (3) está positivamente relacionada a melhores condições de saúde, em relação aos desocupados. De forma geral, observa-se que o mercado de trabalho melhora o estado de saúde individual, dado os demais controles. 
Tabela 3 - Resultados do modelo probit ordenado para o estado de saúde

\begin{tabular}{|c|c|c|c|c|c|c|}
\hline Variáveis & Modelo sem & correção (1) & $\begin{array}{l}\text { Modelo } \\
\text { multi }\end{array}$ & $\begin{array}{l}\text { correção } \\
\text { ial (2) }\end{array}$ & $\begin{array}{r}\text { Modelo c } \\
\text { mipa }\end{array}$ & $\begin{array}{l}\text { orreção se- } \\
\text { trica (3) }\end{array}$ \\
\hline & Homem & Mulher & Homem & Mulher & Homem & Mulher \\
\hline Migração & $\begin{array}{l}0,0031 \\
(0,0111)\end{array}$ & $\begin{array}{c}-0,0434^{\star \star \star} \\
(0,0090)\end{array}$ & $\begin{array}{l}-0,1430 \\
(0,1540)\end{array}$ & $\begin{array}{c}-0,4850^{\star \star \star} \\
(0,1010)\end{array}$ & $\begin{array}{l}-0,0639 \\
(0,1050)\end{array}$ & $\begin{array}{c}-0,3560^{\star \star \star} \\
(0,0050)\end{array}$ \\
\hline Idade & $\begin{array}{c}-0,0468^{* * *} \\
(0,0011)\end{array}$ & $\begin{array}{c}-0,0452^{\star * *} \\
(0,0008)\end{array}$ & $\begin{array}{c}-0,0468^{* * *} \\
(0,0011)\end{array}$ & $\begin{array}{c}-0,0454^{\star * *} \\
(0,0008)\end{array}$ & $\begin{array}{c}-0,0574^{* * *} \\
(0,0013)\end{array}$ & $\begin{array}{c}-0,0421^{* * *} \\
(0,0013)\end{array}$ \\
\hline Idade ao quadrado & $\begin{array}{l}0,0003^{\star \star *} \\
(1,32 \mathrm{e}-05)\end{array}$ & $\begin{array}{l}0,0003^{\star * *} \\
(9,76 e-06)\end{array}$ & $\begin{array}{l}0,0003^{* * *} \\
(1,32 e-05)\end{array}$ & $\begin{array}{c}0,0003^{* * *} \\
(9,76 \mathrm{e}-06)\end{array}$ & $\begin{array}{l}0,0004^{\star * *} \\
(2,36 \mathrm{e}-05)\end{array}$ & $\begin{array}{l}0,0002^{* * *} \\
(1,05 e-05)\end{array}$ \\
\hline Preta & $\begin{array}{c}-0,0494^{\star \star \star} \\
(0,0160)\end{array}$ & $\begin{array}{c}-0,1370^{\star \star \star} \\
(0,0131)\end{array}$ & $\begin{array}{c}-0,0484^{\star \star \star} \\
(0,0160)\end{array}$ & $\begin{array}{c}-0,1350^{\star \star *} \\
(0,0131)\end{array}$ & $\begin{array}{c}-0,0476^{\star \star *} \\
(0,0182)\end{array}$ & $\begin{array}{c}-0,1310^{\star \star \star} \\
(0,0140)\end{array}$ \\
\hline Amarela & $\begin{array}{l}-0,0122 \\
(0,0567)\end{array}$ & $\begin{array}{c}-0,1660^{* * *} \\
(0,0447)\end{array}$ & $\begin{array}{l}-0,0115 \\
(0,0567)\end{array}$ & $\begin{array}{c}-0,1670^{* * *} \\
(0,0447)\end{array}$ & $\begin{array}{l}-0,0956 \\
(0,0750)\end{array}$ & $\begin{array}{c}-0,1650^{* * *} \\
(0,0502)\end{array}$ \\
\hline Parda & $\begin{array}{c}-0,0664^{* * *} \\
(0,0096)\end{array}$ & $\begin{array}{c}-0,0974^{\star * *} \\
(0,0076)\end{array}$ & $\begin{array}{c}-0,0664^{\star \star *} \\
(0,0096)\end{array}$ & $\begin{array}{c}-0,0974^{* * *} \\
(0,0076)\end{array}$ & $\begin{array}{c}-0,0515^{\star * *} \\
(0,0119)\end{array}$ & $\begin{array}{c}-0,0643^{\star * *} \\
(0,0085)\end{array}$ \\
\hline Indígena & $\begin{array}{c}-0,2640^{* * *} \\
(0,0701)\end{array}$ & $\begin{array}{l}-0,1060^{*} \\
(0,0550)\end{array}$ & $\begin{array}{c}-0,2650^{* * *} \\
(0,0701)\end{array}$ & $\begin{array}{l}-0,1080^{\star *} \\
(0,0550)\end{array}$ & $\begin{array}{c}-0,2940^{* * *} \\
(0,0779)\end{array}$ & $\begin{array}{l}-0,1060^{*} \\
(0,0563)\end{array}$ \\
\hline 1 a 4 anos de estudo & $\begin{array}{l}0,1060^{\star * *} \\
(0,0145)\end{array}$ & $\begin{array}{c}0,0496^{\star * *} \\
(0,0121)\end{array}$ & $\begin{array}{l}0,1070^{\star \star *} \\
(0,0145)\end{array}$ & $\begin{array}{l}0,0520^{\star * *} \\
(0,0121)\end{array}$ & $\begin{array}{l}0,1520^{\star \star *} \\
(0,0521)\end{array}$ & $\begin{array}{l}0,1010^{\star * *} \\
(0,0148)\end{array}$ \\
\hline 5 a 8 anos de estudo & $\begin{array}{c}0,2350^{\star * *} \\
(0,0154)\end{array}$ & $\begin{array}{l}0,1770^{\star * \star} \\
(0,0127)\end{array}$ & $\begin{array}{l}0,2350^{\star * *} \\
(0,0154)\end{array}$ & $\begin{array}{c}0,1790^{\star \star \star} \\
(0,0127)\end{array}$ & $\begin{array}{l}0,2820^{* * *} \\
(0,0656)\end{array}$ & $\begin{array}{l}0,2310^{* * *} \\
(0,0183)\end{array}$ \\
\hline 9 anos ou mais de estudo & $\begin{array}{l}0,4030^{\star \star *} \\
(0,0161)\end{array}$ & $\begin{array}{c}0,3850^{\star \star *} \\
(0,0130)\end{array}$ & $\begin{array}{c}0,4030^{\star \star *} \\
(0,0161)\end{array}$ & $\begin{array}{c}0,3860^{\star \star *} \\
(0,0130)\end{array}$ & $\begin{array}{l}0,5080^{\star \star *} \\
(0,0747)\end{array}$ & $\begin{array}{l}0,4510^{\star * *} \\
(0,0228)\end{array}$ \\
\hline Ocupação 2 & $\begin{array}{l}0,3980^{\star \star *} \\
(0,0129)\end{array}$ & $\begin{array}{c}0,1740^{\star * *} \\
(0,0101)\end{array}$ & $\begin{array}{c}0,3980^{* * *} \\
(0,0129)\end{array}$ & $\begin{array}{c}0,1740^{\star \star \star} \\
(0,0101)\end{array}$ & $\begin{array}{c}0,3480^{* * *} \\
(0,0507)\end{array}$ & $\begin{array}{c}0,0273 \\
(0,0192)\end{array}$ \\
\hline Ocupação 3 & $\begin{array}{l}0,3760^{\star \star \star} \\
(0,0235)\end{array}$ & $\begin{array}{c}0,2510^{\star \star *} \\
(0,0288)\end{array}$ & $\begin{array}{c}0,3760^{\star \star \star} \\
(0,0235)\end{array}$ & $\begin{array}{c}0,2470^{\star \star *} \\
(0,0288)\end{array}$ & $\begin{array}{l}0,4190^{\star \star *} \\
(0,0338)\end{array}$ & $\begin{array}{l}0,2510^{\star * *} \\
(0,0335)\end{array}$ \\
\hline Ocupação 4 & $\begin{array}{l}0,3010^{\star * *} \\
(0,0137)\end{array}$ & $\begin{array}{c}0,0620^{\star * *} \\
(0,0121)\end{array}$ & $\begin{array}{c}0,3020^{\star \star *} \\
(0,0138)\end{array}$ & $\begin{array}{l}0,0582^{\star \star *} \\
(0,0122)\end{array}$ & $\begin{array}{l}0,3200^{\star \star *} \\
(0,0216)\end{array}$ & $\begin{array}{l}0,0437^{* * *} \\
(0,0123)\end{array}$ \\
\hline
\end{tabular}




\begin{tabular}{|c|c|c|c|c|c|c|}
\hline \multirow[t]{2}{*}{ Ocupação 5} & $0,2460^{\star \star \star}$ & 0,0090 & $0,2460^{\star \star \star}$ & 0,0080 & $0,3050^{\star \star \star}$ & $0,0495^{\star \star *}$ \\
\hline & $(0,0290)$ & $(0,0180)$ & $(0,0290)$ & $(0,0180)$ & $(0,0200)$ & $(0,0106)$ \\
\hline \multirow[t]{2}{*}{ Ocupação 6} & $0,2560^{\star \star \star}$ & $0,0501^{\star \star \star}$ & $0,2560^{\star \star \star}$ & $0,0473^{\star \star \star}$ & $0,4620^{\star \star \star}$ & $0,1950^{\star \star \star}$ \\
\hline & $(0,0140)$ & $(0,0101)$ & $(0,0140)$ & $(0,0101)$ & $(0,0144)$ & $(0,0110)$ \\
\hline \multirow[t]{2}{*}{$1 \mathrm{SM}$} & $-0,1150^{\star \star \star}$ & $-0,0037$ & $-0,1160^{\star * *}$ & $-0,0026$ & $-0,1820^{\star}$ & $0,0825^{\star \star *}$ \\
\hline & $(0,0378)$ & $(0,0248)$ & $(0,0378)$ & $(0,0248)$ & $(0,1070)$ & $(0,0267)$ \\
\hline \multirow[t]{2}{*}{ Entre 2 e $3 \mathrm{SM}$} & 0,0134 & $0,1460^{\star \star \star}$ & 0,0136 & $0,1470^{\star \star \star}$ & $-0,0688$ & $0,1930^{\star \star *}$ \\
\hline & $(0,0385)$ & $(0,0257)$ & $(0,0385)$ & $(0,0257)$ & $(0,1090)$ & $(0,0304)$ \\
\hline \multirow[t]{2}{*}{ Entre 3 e 4 SM } & $0,1720^{\star \star *}$ & $0,3170^{\star * *}$ & $0,1720^{\star \star \star}$ & $0,3200^{* * *}$ & 0,0606 & $0,3360^{* * *}$ \\
\hline & $(0,0404)$ & $(0,0278)$ & $(0,0404)$ & $(0,0278)$ & $(0,1470)$ & $(0,0376)$ \\
\hline \multirow[t]{2}{*}{ Entre 4 e $5 \mathrm{SM}$} & $0,2350^{\star \star \star}$ & $0,4420^{\star \star \star}$ & $0,2360^{\star \star \star}$ & $0,4440^{\star \star \star}$ & 0,0942 & $0,4670^{\star \star \star}$ \\
\hline & $(0,0433)$ & $(0,0306)$ & $(0,0433)$ & $(0,0306)$ & $(0,1430)$ & $(0,0420)$ \\
\hline \multirow[t]{2}{*}{ Entre 5 e $6 \mathrm{SM}$} & $0,3030^{\star \star \star}$ & $0,5540^{\star \star \star}$ & $0,3030^{\star \star \star}$ & $0,5540^{\star \star \star}$ & 0,0956 & $0,5640^{\star \star \star}$ \\
\hline & $(0,0461)$ & $(0,0346)$ & $(0,0461)$ & $(0,0346)$ & $(0,1240)$ & $(0,0492)$ \\
\hline \multirow[t]{2}{*}{ Mais de $6 \mathrm{SM}$} & $0,4350^{\star \star \star}$ & $0,6970^{\star \star \star}$ & $0,4350^{\star \star \star}$ & $0,6950^{\star \star \star}$ & $0,2980^{\star \star}$ & $0,7330^{\star \star \star}$ \\
\hline & $(0,0420)$ & $(0,0300)$ & $(0,0420)$ & $(0,0299)$ & $(0,1330)$ & $(0,0457)$ \\
\hline \multirow[t]{2}{*}{ Bens de consumo } & $-0,0121$ & 0,0021 & $-0,0119$ & 0,0023 & $-0,0199$ & $-0,0209^{* *}$ \\
\hline & $(0,0099)$ & $(0,0085)$ & $(0,0099)$ & $(0,0085)$ & $(0,0220)$ & $(0,0094)$ \\
\hline \multirow[t]{2}{*}{ Saneamento } & $-0,0126$ & $0,0360^{\star \star \star}$ & $-0,0126$ & $0,0361^{\star \star \star}$ & $-0,0337^{\star \star *}$ & $0,0169^{\star *}$ \\
\hline & $(0,0084)$ & $(0,0069)$ & $(0,0084)$ & $(0,0069)$ & $(0,0116)$ & $(0,0073)$ \\
\hline \multirow[t]{2}{*}{ Área urbana } & $-0,0203$ & $-0,0564^{* * *}$ & $-0,0212$ & $-0,0623^{\star * *}$ & $-0,0029$ & $-0,0179$ \\
\hline & $(0,0129)$ & $(0,0106)$ & $(0,0129)$ & $(0,0107)$ & $(0,0175)$ & $(0,0115)$ \\
\hline \multirow[t]{2}{*}{ Região Metropolitana } & 0,0079 & $0,0449^{\star \star \star}$ & 0,0048 & $0,0341^{\star \star *}$ & $-0,0045$ & $0,0408^{\star \star *}$ \\
\hline & $(0,0109)$ & $(0,0086)$ & $(0,0114)$ & $(0,0088)$ & $(0,0168)$ & $(0,0090)$ \\
\hline Threshold 1 & $\begin{array}{c}-3,5810^{* * *} \\
(0,0544)\end{array}$ & $\begin{array}{c}-3,5660^{\star * *} \\
(0,0402)\end{array}$ & $\begin{array}{c}-3,5940^{\star \star *} \\
(0,0555)\end{array}$ & $\begin{array}{c}-3,5900^{\star \star *} \\
(0,0409)\end{array}$ & Fixo & Fixo \\
\hline \multirow[t]{2}{*}{ Threshold 2} & $-2,7550^{\star \star \star}$ & $-2,8240^{\star * *}$ & $-2,7680^{\star \star \star}$ & $-2,8470^{\star \star \star}$ & $-3,4340^{\star \star \star}$ & $-2,8980^{\star * \star}$ \\
\hline & $(0,0525)$ & $(0,0392)$ & $(0,0537)$ & $(0,0399)$ & $(0,0948)$ & $(0,0232)$ \\
\hline \multirow[t]{2}{*}{ Threshold 3} & $-1,5200^{* * *}$ & $-1,5260^{* * *}$ & $-1,5330^{\star \star *}$ & $-1,5500^{\star * *}$ & $-2,2910^{\star \star *}$ & $-1,7090^{\star * *}$ \\
\hline & $(0,0517)$ & $(0,0386)$ & $(0,0529)$ & $(0,0393)$ & $(0,1980)$ & $(0,0572)$ \\
\hline
\end{tabular}




\begin{tabular}{l|c|c|c|c|c|c} 
cont. & $0,1210^{\star *}$ & $0,0961^{\star *}$ & $0,1080^{\star *}$ & $0,0726^{*}$ & $0,3340^{\star \star *}$ & $0,3740^{\star * *}$ \\
Threshold 4 & $(0,0515)$ & $(0,0384)$ & $(0,0527)$ & $(0,0391)$ & $(0,0580)$ & $(0,0934)$ \\
& $157.818,70$ & $250.029,90$ & $157.817,90$ & $250.031,50$ & $346.580,40$ & $542.150,30$ \\
AIC & $158.326,80$ & $250.561,70$ & 158.326 & $250.563,30$ & $347.356,30$ & $542.981,08$ \\
$\mathrm{BIC}$ & 75.907 & 116.894 & 75.907 & 116.894 & 75.907 & 116.894 \\
\hline
\end{tabular}

Notas: a) ${ }^{* * *}$ significativo a $1 \%$; ** significativo a $5 \%$; * significativo a $10 \%$.

b) Erro-padrão entre parênteses.

Fonte: microdados PNAD 2008. Elaborado pelos autores.

V- Renda monetária: os resultados dos coeficientes devem ser interpretados em relação à variável omitida, neste caso os indivíduos sem rendimento. $\mathrm{O}$ fato de a primeira dummy apresentar um sinal negativo reflete um resultado interessante, qual seja o de que os homens com rendimento de até $\mathrm{R} \$$ 415,00 têm menor probabilidade de apresentarem um estado de saúde melhor. Por outro lado, a dummy que indica rendimentos entre dois e três salários mínimos somente é estatisticamente significativa para as mulheres. Conforme o modelo 3, a variável que captura os salários dos homens entre dois e seis salários mínimos não se mostrou significativa. Para as demais classes de salário mínimo, o impacto da renda é positivo e estatisticamente significativo. Nota-se que há uma alta correlação entre a condição de saúde e o rendimento monetário, isto é, o aumento da renda está associado a uma redução da probabilidade do indivíduo apresentar um estado de saúde muito ruim e a um aumento da probabilidade de apresentar um estado de saúde muito bom, conforme a Figura 2. 
Figura 2 - Relação entre probabilidade dos estados de saúde e renda monetária
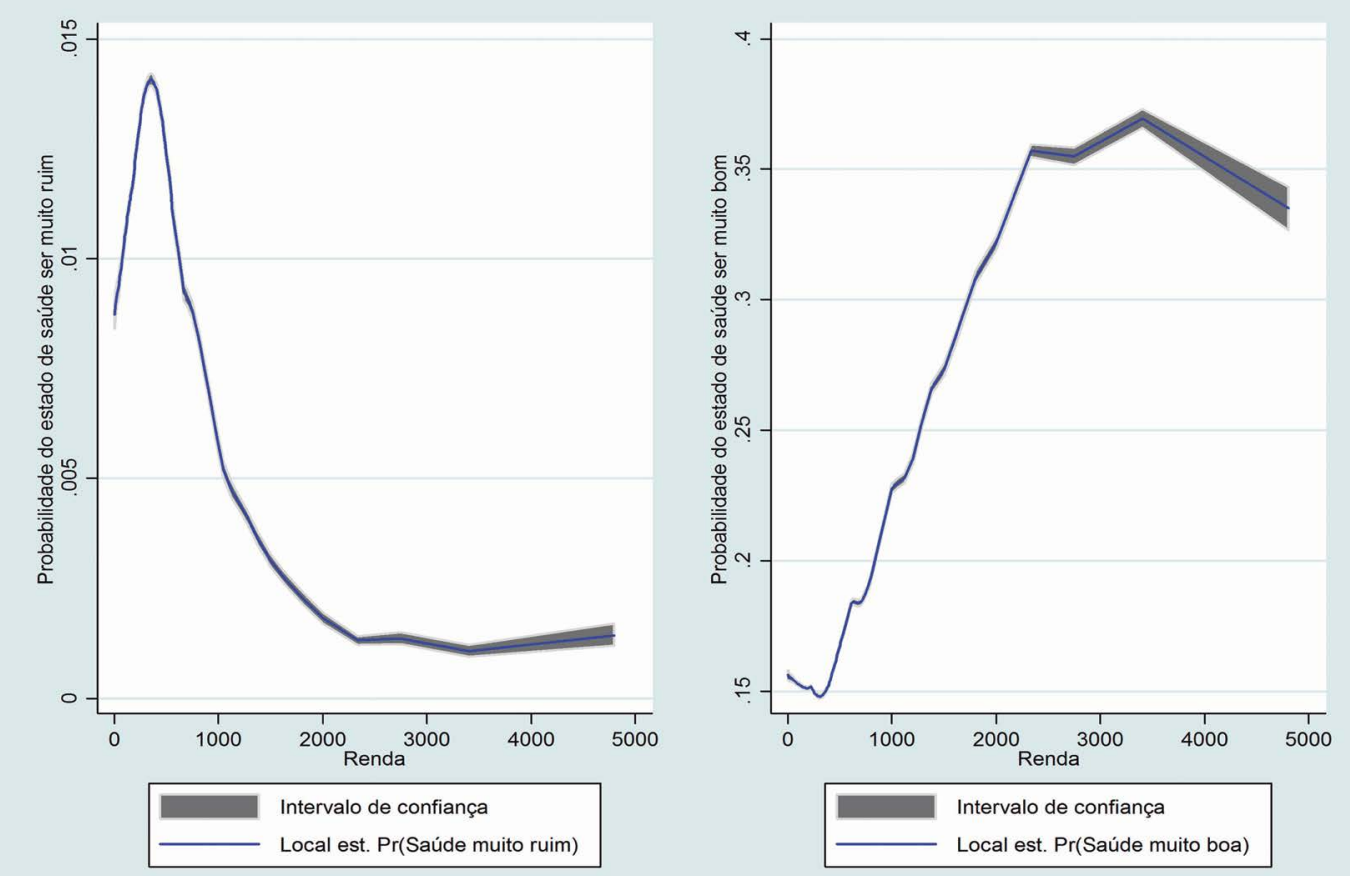

VI- Bens de consumo: o indicador obtido por meio de análise fatorial com a extração de dois componentes principais não se mostrou significativo em nenhum modelo estimado, com exceção do modelo 3 para as mulheres, porém, apresentou um sinal não esperado.

VII- Saneamento: as características do setor de saúde e as mudanças quanto à estrutura etária e ao padrão de mortalidade e morbidade que diversos países têm passado apresenta consequências sobre custos e utilização de serviços médicos e ambulatoriais (RODRIGUES et al., 2010). Além disso, as transformações nos sistemas de saúde - reorganização dos modelos de atenção - e a incorporação de novas tecnologias, dentre outros fatores, têm alterado os padrões de utilização destes serviços. A composição desses múltiplos efeitos pode ser reunida em uma variável, a saber, o saneamento básico. Essa variável apresenta informações a respeito da qualidade de vida das famílias que dependem mais das políticas públicas que da própria renda e permite investigar seu impacto sob as condições de saúde. O indicador mostrou-se significativo a $1 \%$ e apresentou o sinal esperado para o modelo que analisa o estado de saúde das mulheres. Dessa forma, melhorias das 
condições do setor de saúde convertem-se em uma melhor percepção do estado de saúde autorreportado.

VIII-Variáveis geográficas: as variáveis para capturar diferenças geográficas indicou que as mulheres residentes em áreas urbanas apresentam menor probabilidade de ter um nível de saúde adequado, enquanto as residentes em regiões metropolitanas apresentam o resultado inverso. Esse resultado pode estar refletindo o fato de que apesar das localidades urbanas apresentarem, em média, mais recursos e relativamente mais insumos do que as áreas rurais, esse fator não gera um valor adicionado ao estoque de saúde individual. Por outro lado, as regiões metropolitanas apresentam grandes conglomerações relacionadas ao setor de saúde. A utilização de serviços de saúde é resultado da interação entre a necessidade do indivíduo e a sua decisão de procurar cuidados médicos. Os determinantes dessa utilização estão associados às características dos sujeitos, do sistema de saúde e ao padrão de prática médica (RIBEIRO, 2005). Arrow (1963) destaca que o setor de saúde dispõe de características particulares que justificam seu estudo como um ramo destacado da economia. Dentre essas particularidades, o autor cita: a imprevisibilidade e a irregularidade da demanda pelos serviços, uma vez que o indivíduo não sabe quando e com que frequência necessitará de atenção médica; o comprometimento da racionalidade do consumidor na escolha de bens e serviços, visto que a demanda ocorre em uma situação anormal de doença; a incapacidade de recorrer às experiências anteriores para mitigar algum risco para o paciente. Por esses motivos, os indivíduos que vivem nas regiões metropolitanas estão mais próximos aos centros de saúde e conseguem reduzir a depreciação de seu estado de saúde, o que se reflete sobre sua autoavaliação de saúde.

IX- Migração: a variável principal deste estudo apresentou resultados interessantes. Observa-se que ela é estatisticamente significativa e apresenta um sinal negativo somente no modelo que estima o efeito da migração sobre as condições de saúde para as mulheres. Além disso, o efeito é maior, em módulo, no modelo que corrige o viés de seleção por intermédio do modelo multinomial. Assim, a partir da hipótese de Van Der Vem e Ellis (2000) foi possível observar a presença de uma variante do chamado paradoxo epidemiológico. Isto é, o estado de saúde individual deteriora-se, ao longo do tempo, para as mulheres, refletindo uma situação inversa a que foi proposta por Hull (1979). Isso significa que o efeito líquido da migração é negativo, que, por sua vez, pode impactar de forma negativa a respeito da produtividade e do crescimento econômico. 
Os critérios de informação AIC e BIC sugerem que a especificação mais adequada para o modelo que avalia o impacto da migração sobre a saúde para os homens é aquela que corrige o viés de seleção por meio do modelo multinomial, apesar da variável que capta este ajuste não ser significativa. Para o caso das mulheres ocorre o inverso, isto é, o modelo que não corrige o viés é o mais adequado, o que corrobora a hipótese de Atella e Deb (2013). Assim, observa-se que as duas estratégias empíricas propostas para corrigir o viés de seleção são consistentes e indicam para a mesma direção.

\section{Considerações finais}

O objetivo deste estudo é avaliar o impacto da migração sobre o estado de saúde individual. Esta hipótese foi proposta por Hull (1979), porém o número de estudos que procuram investigá-la é reduzido na literatura internacional e inexistente no âmbito nacional. O processo migratório pode constituir um fator de risco para a saúde, podendo acarretar uma maior vulnerabilidade em relação a problemas de saúde em geral. A migração, ao influenciar a saúde individualmente, altera o nível de produtividade e, portanto, a taxa de crescimento econômico no curto e no longo prazo. Para verificar essa relação, considerou-se que a migração atua por canais distintos sobre a saúde de homens e mulheres (VAN DER VEM; ELLIS, 2000; ATELLA; DEB, 2013). Como enfatizado por Bäckström (2010), a migração pode contribuir diretamente para a deterioração do estado de saúde, por meio de precárias condições de habitabilidade, de alimentação deficiente, de baixos rendimentos e de difíceis e incertas condições de contratação e de segurança no trabalho.

A partir dos microdados da PNAD 2008 foram estimados três modelos, que incorporam o plano amostral da base de dados utilizada. Em primeiro lugar, estimou-se um modelo probit ordenado, considerando o impacto da migração por meio de uma variável dummy. Em segundo lugar, corrigiu-se o viés de seleção da amostra por intermédio de um modelo probit multinomial. Finalmente, considerou-se uma abordagem semiparamétrica para corrigir o mesmo problema sob a estrutura de um modelo probit ordenado. Como a decisão de migrar não é um processo aleatório, foi inserida a noção de clusters para corrigir a precisão das estimativas dos modelos que corrigem o viés de seleção.

Os determinantes socioeconômicos têm ganho espaço no esforço de compreensão da relação entre migração e saúde. Os resultados dos modelos estimados indicaram que o viés de seleção é relevante no caso dos homens, enquanto o modelo que não corrige o viés é o mais adequado para as mulheres. Além disso, nota-se que 
as duas estratégias empíricas propostas para corrigir o viés de seleção são consistentes e indicam para a mesma direção. Esses resultados corroboram a hipótese de Atella e Deb (2013). Melhores status saúde estão associados, de forma geral, a maiores níveis de educação formal e de renda. Assim, os principais resultados indicam que os custos da migração superam os benefícios, como encontrado por Gibson e McKenzie (2007) para Tonga e Nova Zelândia. 


\title{
Sesgo de selección, migración y salud
}

\section{Resumen}

El objetivo del estudio es evaluar el impacto de la migración en el estado de salud individual. Esta hipótesis propuesta por Hull (1979) se pondrá a prueba a partir de tres estrategias empíricas: i) un modelo probit ordenado teniendo en cuenta el impacto de la migración a través de una variable dummy; ii) un modelo probit multinomial para corregir el sesgo de selección; y iii) el método de estimación semiparamétrica propuesto por De Luca y Perotti (2011). Los resultados de los modelos estimados indican que el sesgo de selección es relevante en el caso de los hombres, mientras que el modelo que no corrige el sesgo es más adecuado para las mujeres. La probabilidad de transición de un nivel de muy mala salud a un nivel que se considera muy bueno se asocia com mayores niveles de educación formal e ingresos.

Palabras clave: Migración. La salud. El sesgo de selección.

\section{Selection bias, migration and health}

\begin{abstract}
The objective of the study is to evaluate the impact of migration on individual health status. This hypothesis proposed by Hull (1979) will be tested from three empirical strategies: i) an ordered probit model considering the impact of migration through a dummy variable; ii) a multinomial probit model to correct for selection bias, and iii) semiparametric estimation method proposed by De Luca and Perroti (2011). The results of the estimated models indicated that the selection bias is relevant in the case of men, while the model that does not correct the bias is most suitable for women. The probability of transition from one level of very bad health to a level considered very good is associated with higher levels of formal education and income.
\end{abstract}

Keywords: Migration. Health. Selection bias.

Classificação JEL: C25, C14, O15, I10. 


\section{Notas}

1 Para ver mais detalhes observar Cameron e Trivedi (2005).

2 Para ver mais detalhes observar Cameron e Trivedi (2005).

3 É a maneira mais confiável e precisa para simular probabilidades normais multivariadas para a estimação clássica.

4 O estimador é obtido, ajustando localmente um polinômio de grau $p$ aos dados por mínimos quadrados ponderados e aproxima o intervalo de confiança por meio de uma transformação logística.

\section{Referências}

ANSON, J. The migrant mortality advantage: a 10 month followup of the Brussels population. European Journal of Population, v. 20, p. 191-218, 2004.

ARROW, K. J. Uncertainty and welfare economics of medical care. American Economic Review, Pittsburgh, v. 53, n. 5 p. 941-973, Dec. 1963.

ATELLA, V.; DEB, P. Gender Differences in Long Term Health Outcomes of Internal Migrants in Italy. CEIS Tor Vergata. Research Paper Series n. 269, 2013.

BÄCKSTRÖM, B. O acesso à saúde e os factores de vulnerabilidade na população imigrante. Alicerces, Instituto politécnico de Lisboa, v. 3, n. 3, p. 79-90, abr. 2010.

BORJAS, G. J.; BRONARS, S. G.; TREJO, S. J. Self-Selection and Internal Migration in the United States. Journal of Urban Economics, v. 32, p. 159-185, set. 1992.

BRITO, F. População, espaço e economia: uma perspectiva histórica. Tese de Doutorado. Programa de Pós-Graduação em Demografia, Cedeplar/UFMG. Belo Horizonte, 1997.

Brasil, final do século: a transição para um novo padrão migratório. In: CARLEIAL, A. (org.). Transições migratórias. Fortaleza: Inplance, 2002.

CAMERON, A.; TRIVEDI, P. Microeconometrics: Methods and Applications. Cambridge: University Press, 2005.

CHISWICK, B. R. The Effect of Americanization on the Earnings of Foreign-Born Men. Journal of Political Economy, The University of Chicago, v. 86, p. 897-921, 1978.

DAHL, G. B. Mobility and the Return to Education: Testing a Roy Model with Multiple Markets. Econometrica, v. 70, n. 6, p. 2367-2420, nov. 2002.

DE LUCA, G. SNP and SML estimation of univariate and bivariate binary choice models. The Stata Journal, v. 8, p. 190-220, 2008.

DE LUCA, G.; PEROTTI, V. Estimation of ordered response models with sample selection. The Stata Journal, v. 11, p. 213-39, 2011.

FERANIL, A. B. Anaemia among migrant and non-migrant mothers in disadvantaged areas in the Visayas, the Philippines. Migration and health in Asia, p. 100-115, 2005.

FRANK, R.; HUMMER, R. A. The Other Side of the Paradox: The Risk of Low Birth Weight among Infants of Migrant and Nonmigrant Households within Mexico. International Migration Review, v. 36, p. 746-765, set. 2002. 
GALLANT A. R.; NYCHKA, D. W. Semi-nonparametric maximum likelihood estimation. Econometrica, v. 55, p. 363-390, mar. 1987.

GEWEKE, J. Evaluating the Accuracy of Sampling-based Approaches to the Calculation of Posterior Moments (with Discussion). In: BERNARDO, J.; BERGER, J.; DAWID, A. P.; SMITH, A. F. M. (Eds.). Bayesian Statistics. Oxford: Oxford University Press, 1992.

GIBSON, J.; MCKENZIE, D. The Impact of an Ex-Ante Job Other Requirement on Labor Migration: The New Zealand-Tongan Experience. In: OZDEN, C.; SCHIFF, M. (Eds.). International Migration, Economic Development and Policy. World Bank, Washington D.C., 2007.

GROSSMAN, M. On the concept of health and the demand for health. Journal of Political Economy, The University of Chicago Press, v. 80, n. 2, p. 223-255, mar. 1972.

HAJIVASSILIOU, V. A.; MCFADDEN, D. A Simulation Estimation Analysis of the External Debt Crises of Developing Countries. Journal of Applied Econometrics, New Haven, USA, v. 9, p. 109-131, set. 1994.

HECKMAN, J. Sample Selection Bias as a Specification Error. Econometrica, v. 47, n. 1, p. 153161, Jan. 1979.

HULL, D. Migration, adaptation, and illness: a review. Social Science and Medicine, v. 13A, p. 25-36, Jan. 1979.

KANAIAUPUNI, S. M.; DONATO, K. M. Migradollars and mortality: The effects of migration on infant survival in Mexico. Demography, v. 36, n. 3, p. 339-353, Ago. 1999.

KEANE, M. P. A Computationally Practical Simulation Estimator for Panel Data. Econometrica, The Econometric Society, v. 62, p. 95-116, Jan. 1994.

LIMA, A. C. C.; SIMÕES, R.; OLIVEIRA, A. M. H. C. Caracterização dos padrões migratórios brasileiros no período 1980-2010. In: ENCONTRO NACIONAL DE ESTUDOS POPULACIONAIS, XVIII, Anais...Águas de Lindóia/SP, 2012.

LU, Y. Test of the 'healthy migrant hypothesis': A longitudinal analysis of health selectivity of internal migration in Indonesia. Social Science and Medicine, v. 67, p. 1331-1339, Out. 2008.

MADDALA, G. S. Limited Dependented Variables. Cambridge: University Press, 1984.

MARMOT, M. G.; ADELSTEIN, M. A.; BULUSU, L. Immigrant mortality in England and Wales 1970-1978. OPCS studies of medical and population subjects, 47. London: HMSO, 1984a.

Lessons from the study of immigrant mortality. Lancet, v. 323, p. 1455-1457, nov. 1984b.

NASCIMENTO SILVA, P. L.; PESSOA, D. G. C.; LILA, M. F. Statistical analysis of data from PNAD: incorporating the sample design. Ciência \& Saúde Coletiva, Rio de Janeiro, v. 7, n. 4, p. 659-670, 2002.

POWELL, J. Semiparametric Methods. In: HECKMAN, J.; MCFADDEN, D. (Eds). Handbook of Econometrics, v. 4. Amsterdam: North-Holland, 1997.

RIBEIRO, E. P.; BASTOS, V. M. Viés de Seleção, Retornos à Educação e Migração no Brasil. In: Anais do XXVI EBE. Rio de Janeiro: SBE, 2004.

RIBEIRO, M. M. Utilização de serviços de saúde no Brasil: uma investigação do padrão etário por sexo e cobertura por plano de saúde. 2005. 100 f. Dissertação (Mestrado em Economia), UFMG / CEDEPLAR, Belo Horizonte, 2005. 
RODRIGUES, C. G.; ANDRADE, M. V.; QUEIROZ, B. L; MACHADO, C. J. Análise de taxas de utilização de serviços de saúde temporalmente: elementos para a formulação de um estudo. UFMG/Cedeplar, 2010. 13p. (Texto para discussão; 404).

SANTOS JR., E. R.; FERREIRA, P. C.; MENEZES-FILHO, N. Migração, Seleção e Diferenças Regionais de Renda no Brasil. Pesquisa e Planejamento Econômico, v. 35, n. 3, p. 299-331, 2005.

SIMÕES, R.; AMARAL, P. V. Interiorização e Novas Centralidades Urbanas: Uma Visão Prospectiva para o Brasil. Economia, Brasília, v. 12, n. 3, p. 553-579, 2011.

SILVA, T. F. B.; SILVEIRA NETO, R. M. Migração e seleção no Brasil: evidências para o decênio 1993-2003. In: Anais do X Encontro Regional de Economia, Fortaleza, 2005.

VAN DER VEN, W. P. M. M.; ELLIS, R. P. Risk adjustment in competitive health plan markets. In.: CULYER, A. J.; NEWHOUSE, J. P. (Eds.). Handbook of Health Economics. Amsterdam: North-Holland, 2000.

WHO. Migration and health in the European Union. New York: McGraw Hill, 2009.

WHO. How health systems can address health inequities linked to migration and ethnicity. Copenhagen: WHO Regional Office for Europe, 2010.

WOOLDRIDGE, J. M. Econometric Analysis of Cross Section and Panel Data. Cambridge: The MIT Press, 2000. 
Anexo: Tabela 1 - Estatísticas descritivas da amostra

\begin{tabular}{|c|c|c|c|c|c|c|c|c|}
\hline \multirow{2}{*}{ Variáveis } & \multicolumn{4}{|c|}{ Homens } & \multicolumn{4}{|c|}{ Mulheres } \\
\hline & Média & $\mathrm{DP}^{1}$ & Min & Max & Média & DP & Min & Max \\
\hline Saúde muito ruim & 0,00795 & 0,0888 & 0 & 1 & 0,0109 & 0,1040 & 0 & 1 \\
\hline Saúde ruim & 0,03670 & 0,1880 & 0 & 1 & 0,0406 & 0,1970 & 0 & 1 \\
\hline Saúde regular & 0,20400 & 0,4030 & 0 & 1 & 0,2530 & 0,4350 & 0 & 1 \\
\hline Saúde boa & 0,51900 & 0,5000 & 0 & 1 & 0,5130 & 0,5000 & 0 & 1 \\
\hline Saúde muito boa & 0,23300 & 0,4220 & 0 & 1 & 0,1820 & 0,3860 & 0 & 1 \\
\hline Idade & 39,36000 & 18,6800 & 0 & 107 & 40,0700 & 18,1300 & 0 & 108 \\
\hline Cor 1 & 0,46800 & 0,4990 & 0 & 1 & 0,4860 & 0,5000 & 0 & 1 \\
\hline Cor 2 & 0,07950 & 0,2700 & 0 & 1 & 0,0758 & 0,2650 & 0 & 1 \\
\hline Cor 3 & 0,00577 & 0,0757 & 0 & 1 & 0,0066 & 0,0809 & 0 & 1 \\
\hline Cor 4 & 0,44300 & 0,4970 & 0 & 1 & 0,4280 & 0,4950 & 0 & 1 \\
\hline Cor 5 & 0,00357 & 0,0596 & 0 & 1 & 0,0032 & 0,0566 & 0 & 1 \\
\hline Educação 1 & 0,13100 & 0,3380 & 0 & 1 & 0,1180 & 0,3220 & 0 & 1 \\
\hline Educação 2 & 0,24600 & 0,4310 & 0 & 1 & 0,2400 & 0,4270 & 0 & 1 \\
\hline Educação 3 & 0,25000 & 0,4330 & 0 & 1 & 0,2440 & 0,4300 & 0 & 1 \\
\hline Educação 4 & 0,37300 & 0,4840 & 0 & 1 & 0,3980 & 0,4900 & 0 & 1 \\
\hline Ocupação 1 & 0,02190 & 0,1460 & 0 & 1 & 0,5650 & 0,4960 & 0 & 1 \\
\hline Ocupação 2 & 0,32600 & 0,4690 & 0 & 1 & 0,0352 & 0,1840 & 0 & 1 \\
\hline Ocupação 3 & 0,04140 & 0,1990 & 0 & 1 & 0,0149 & 0,1210 & 0 & 1 \\
\hline Ocupação 4 & 0,18900 & 0,3920 & 0 & 1 & 0,0877 & 0,2830 & 0 & 1 \\
\hline Ocupação 5 & 0,14300 & 0,3500 & 0 & 1 & 0,1310 & 0,3380 & 0 & 1 \\
\hline Ocupação 6 & 0,27800 & 0,4480 & 0 & 1 & 0,1660 & 0,3720 & 0 & 1 \\
\hline Sem remuneração & 0,01340 & 0,1150 & 0 & 1 & 0,0172 & 0,1300 & 0 & 1 \\
\hline $1 \mathrm{SM}$ & 0,50200 & 0,5000 & 0 & 1 & 0,5520 & 0,4970 & 0 & 1 \\
\hline Entre 2 e $3 \mathrm{SM}$ & 0,26100 & 0,4390 & 0 & 1 & 0,2420 & 0,4280 & 0 & 1 \\
\hline Entre 3 e $4 \mathrm{SM}$ & 0,09280 & 0,2900 & 0 & 1 & 0,0802 & 0,2720 & 0 & 1 \\
\hline Entre 4 e $5 \mathrm{SM}$ & 0,04410 & 0,2050 & 0 & 1 & 0,0387 & 0,1930 & 0 & 1 \\
\hline Entre 5 e $6 \mathrm{SM}$ & 0,02520 & 0,1570 & 0 & 1 & 0,0212 & 0,1440 & 0 & 1 \\
\hline Mais de $6 \mathrm{SM}$ & 0,06160 & 0,2410 & 0 & 1 & 0,0486 & 0,2150 & 0 & 1 \\
\hline Bens de consumo & $-0,40100$ & 0,4120 & $-2,480$ & 0,760 & $-0,3930$ & 0,3820 & $-2,480$ & 0,760 \\
\hline Saneamento & 0,67200 & 0,6100 & $-2,037$ & 2,172 & 0,7200 & 0,5780 & $-2,037$ & 2,172 \\
\hline Área urbana & 0,80000 & 0,4000 & 0 & 1 & 0,8390 & 0,3670 & 0 & 1 \\
\hline Região Metropolitana & 0,29200 & 0,4550 & 0 & 1 & 0,2990 & 0,4580 & 0 & 1 \\
\hline
\end{tabular}

Notas: ${ }^{1}$ DP é o desvio-padrão.

Fonte: Microdados PNAD 2008. Elaborado pelos autores. 